\title{
Synergistic inhibition of hepatocellular carcinoma growth and hepatocarcinogenesis by combination of 5-fluorouracil and angiotensin-converting enzyme inhibitor via anti-angiogenic activities
}

\author{
KOJI YANASE, HITOSHI YOSHIJI, YASUHIDE IKENAKA, RYUICHI NOGUCHI, MITSUTERU KITADE, \\ KOSUKE KAJI, JUNICHI YOSHII, TADASHI NAMISAKI, MASAHARU YAMAZAKI, KIYOSHI ASADA, \\ TATSUHIRO TSUJIMOTO, TAKEMI AKAHANE, MASAHITO UEMURA and HIROSHI FUKUI
}

Third Department of Internal Medicine, Nara Medical University, Nara, Japan

Received October 17, 2006; Accepted November 22, 2006

\begin{abstract}
Therapies aimed at destruction of the tumor vasculature are now recognized as a promising approach against cancer, and it has been reported that the combination treatment with an angiogenic inhibitor and conventional chemotherapeutic drug exerted synergistic anti-cancerous effects. We previously reported that the clinically used angiotensin-converting enzyme inhibitor (ACE-I) exerted potent-anti-angiogenic activities. The aim of our current study was to examine the combined effect of ACE-I and 5-fluorouracil (5-FU), which is widely used for hepatogastrointestinal tumors, on hepatocellular carcinoma (HCC) growth and hepatocarcinogenesis. When used individually at low doses, neither 5-FU nor ACE-I exerted significant inhibitory effects on the HCC growth. However, the combination treatment of 5-FU and ACE-I showed a potent inhibitory effect on HCC growth along with suppression of neovascularization in the tumor. The expression level of the vascular endothelial growth factor, a potent angiogenic factor, was also suppressed, almost in conjunction with tumor growth inhibition. Furthermore, 5-FU and ACE-I treatment resulted in a marked increase of apoptosis in the tumor. In the hepatocarcinogenesis model, the combination treatment with 5-FU and ACE-I also showed a marked inhibitory effect on the development of preneoplastic lesions. The in vitro study demonstrated that this combination treatment inhibited endothelial cell tubular formation. Collectively, the combination treatment of 5-FU and ACE-I exerted a marked synergistic inhibitory effect on HCC growth via suppression of angiogenesis. This regimen also showed a chemopreventive effect against hepatocarcinogenesis. Since
\end{abstract}

Correspondence to: Dr Hitoshi Yoshiji, Third Department of Internal Medicine, Nara Medical University, Shijo-cho 840, Kashihara, Nara 634-8522, Japan

E-mail: yoshijih@naramed-u.ac.jp

Key words: angiogenesis, angiotensin-converting enzyme, 5fluorouracil, hepatocarcinogenesis, hepatocellular carcinoma both 5-FU and ACE-I are widely used in clinical practice, this combination therapy may be an effective new therapeutic strategy against $\mathrm{HCC}$ in the future.

\section{Introduction}

Any solid tumor that has not acquired its new own blood supply can not grow to more than only a few millimeters in size, including hepatocellular carcinoma (HCC) $(1,2)$. Therapies targeting the tumor vessels have proven very successful for cancer treatment in experimental models (3). Anti-angiogenic therapy is still under investigation all over the world, including the use of gene therapy, anti-angiogenic recombinant proteins, monoclonal antibodies, and various drugs (4). However, some recent studies have suggested that a treatment using a single anti-angiogenic agent may not be sufficient to completely inhibit the tumor angiogenesis $(5,6)$. The use of anti-angiogenic agents as monotherapy in treating patients with advanced cancer has not yet shown a significant efficacy $(2,4)$. The limitations of anti-angiogenic monotherapy in this setting were in fact predicted by pre-clinical studies on the angiogenesis inhibitors endostatin and angiostatin. It has been reported that the combination treatment of anti-angiogenic agents, such as endostatin and angiostatin, exerted a synergistic inhibitory effect on the tumor development and angiogenesis $(4,7)$. Also, it is increasingly likely that cancer therapy will need to be combinatorial (8). Much pre-clinical evidence indicates that combining anti-angiogenic agents with a conventional chemotherapeutic agent therapy results in an additive or even synergistic anti-tumoral effect (9). In February 2004, the US Food and Drug Administration (FDA) approved bevacizumab, a humanized anti-VEGF (vascular endothelial growth factor) monoclonal antibody (VEGF mAb), for treatment of advanced colorectal cancer in combination with 5-fluorouracil (5-FU)based chemotherapy regimens, and this combination treatment exerted a marked survival benefit (8).

$\mathrm{HCC}$ is one of the most common malignancies in the world. The prognosis of HCC is still poor since it develops in conjunction with chronic liver diseases, such as liver cirrhosis (10). Radical operation is the only curative modality for HCC, but it is appropriate in the minority of patients due to limited 
hepatic reserves. Therefore, various palliative therapeutic modalities, such as trans-arterial embolization (TAE), percutaneous radiofrequency ablation (RFA), and orthotopic transplantation, have been employed, but there is still no satisfactory treatment for HCC. One of the characteristic features of HCC in clinical practice is hypervascularity. Several studies have shown that neovascularization and angiogenic factors, such as VEGF, are significantly up-regulated in the human HCC samples $(11,12)$. We previously reported that angiogenesis plays a pivotal role in murine HCC development, and that suppression of VEGF-signaling pathway markedly attenuated the tumor growth (13-15). In addition, some studies have demonstrated that angiogenesis begins at a very early stage when the tumor consists of just 100-300 cells (16). Moreover, several reports have indicated that angiogenesis is involved in the early carcinogenesis step (17-19). A recent study on the endothelial cell markers in the dysplastic lesions of the liver has suggested that in HCC, alterations in the hepatic microcirculation occur at a very early stage of liver carcinogenesis (20). Similar to the HCC growth, we have proved that angiogenesis played a pivotal role in murine hepatocarcinogenesis (21). Collectively, it is very likely that the combination treatment of 5-FU and VEGF mAb also would be a promising approach against HCC. However, no clinical trial with VEGF mAb against HCC is underway. An alternative approach is to employ a clinically available agent, which exerts an anti-angiogenic effect.

We previously reported that the clinically used angiotensin-converting enzyme inhibitor (ACE-I), perindopril (PE), possesses strong anti-angiogenic activity, that it inhibited murine HCC growth (22), and that ACE-I showed a marked suppressive effect against the development of liver enzyme-altered preneoplastic lesions in rats via angiogenesis suppression (23). We also observed that the VEGF expression was significantly suppressed by ACE-I both in vitro and in vivo (22).

In the current study, we examined whether the combination treatment with low doses of 5-FU and ACE-I exerted a synergistic effect on HCC growth and hepatocarcinogenesis. We also sought to clarify the possible mechanism especially in conjunction with alteration of neovascularization and VEGF expression.

\section{Materials and methods}

Compounds and animal treatment. PE (an ACE-I) and 5-FU were generously supplied by Daiichi Pharmaceutical Co. (Tokyo, Japan), and Kyowa-Hakko Inc. (Tokyo), respectively. Male BALB/c mice and SD rats, aged 6 weeks, were purchased from Japan SLC Inc. (Hamamatsu, Shizuoka, Japan) for the study of HCC growth and hepatocarcinogenesis, respectively. They were housed in stainless-steel mesh cages under controlled conditions of temperature $\left(23 \pm 3^{\circ} \mathrm{C}\right)$ and relative humidity $(50 \pm 20 \%)$, with $10-15$ air changes per hour and light illumination for $12 \mathrm{~h}$ a day. The animals were allowed access to food and tap water ad libitum throughout the acclimatization and experimental periods. The murine HCC cell line, BNL. 1 ME A.7R.1 (BNL-HCC) and HUVEC were obtained from the Japanese Cancer Research Resources Bank (Tokyo). The BNL-HCC cells are an adherent, chemically transformed, mouse liver cell line derived from the normal $\mathrm{BALB} / \mathrm{c}$ embryonic liver cell line, BNL CL2 (American Tissue Culture Collection, Manassas, VA; TIB 73) as described previously. (24) To create the allograft model, $1 \times 10^{6}$ of BNL-HCC cells were injected into the flanks of BALB/c mice. The mice were randomly divided into five groups $(n=10$ in each group). Group 1 (G1) consisted of untreated mice that served as the control group. The mice in $\mathrm{G} 2$ and $\mathrm{G} 3$ received $0.5 \mathrm{mg} / \mathrm{kg} /$ day of ACE-I and $5 \mathrm{mg} / \mathrm{kg} / \mathrm{day}$ of 5 -FU by daily gavage, respectively. The combination treatment of 5-FU and ACE-I group was designated as G4. The tumor volume was measured twice a week, and the mice were sacrificed 35 days after the tumor cell implantation. For hepatocarcinogenesis study, the rats were divided into five groups ( $\mathrm{n}=10$ each). The rats in group 1 to group 4 (G1 to G4) received an intraperitoneal (i.p.) injection of $75 \mathrm{mg} / \mathrm{kg}$ of diethylnitrosamine (DEN) weekly for 3 weeks, and then $100 \mathrm{mg} / \mathrm{kg}$ of DEN for 3 weeks followed by phenobarbital (PB) mixed with each diet at a concentration of $0.05 \%$ from week 6 to the final survival as described previously $(25,26)$. The design of the respective group from G1 to G4 was similar to that in the HCC tumor growth experiment. The rats, which received phosphatebuffered saline (PBS) instead of DEN, were examined as a negative control group (G5). All animal procedures were performed according to standard protocols and in accordance with the standard recommendations for the proper care and use of laboratory animals.

Semi-quantitative analysis of CD31 RNA expression in the tumor. To evaluate the expression of CD31 RNA, which is widely used as a neovascularization marker, we performed a semi-quantitative RT-PCR analysis. The tumors, selected in the same way as for measurement of VEGF, were immediately snap-frozen for RNA extraction $(n=5$ in each experimental group), and mRNA was extracted from the pool of tumors. The primer for the mouse CD31 was as follows: sense, 5'TGCTGTTCTATAAGGACGAT-3' (285-304); anti-sense, 5'-CACTCCTGATGGGTTCTGACAG-3' (719-738). PCR was performed at $94^{\circ} \mathrm{C}$ for $1 \mathrm{~min}$, at $53.5^{\circ} \mathrm{C}$ for $1 \mathrm{~min}$, and at $72^{\circ} \mathrm{C}$ for $1 \mathrm{~min}$ for 30 cycles. To prevent genomic DNA contamination, all RNA samples were subjected to DNase I digestion and checked by 30 cycles of PCR to confirm the absence of any amplified DNA. Densitometric analysis was performed by measuring the absorbance of each band with a Fuji BAS 2000 image analyzer (Fuji Co., Tokyo). Glyceraldehyde-3-phosphate dehydrogenase (GAPDH) was used as an internal control.

Measurement of the VEGF expression in the tumor. We measured the VEGF expression level in the tumor. Because a different size of tumor may cause different hypoxic conditions, which strongly induce VEGF (27), five tumors having the same size were chosen from each group. The tumor samples were prepared as described previously (28). After the protein concentration was equalized, the VEGF level was measured with an ELISA kit (R\&D Systems, Minneapolis, MN, USA) in accordance with the supplier's instructions.

Immunohistochemistry. Apoptosis was detected with the DNA fragmentation products that were stained by in situ 3' 


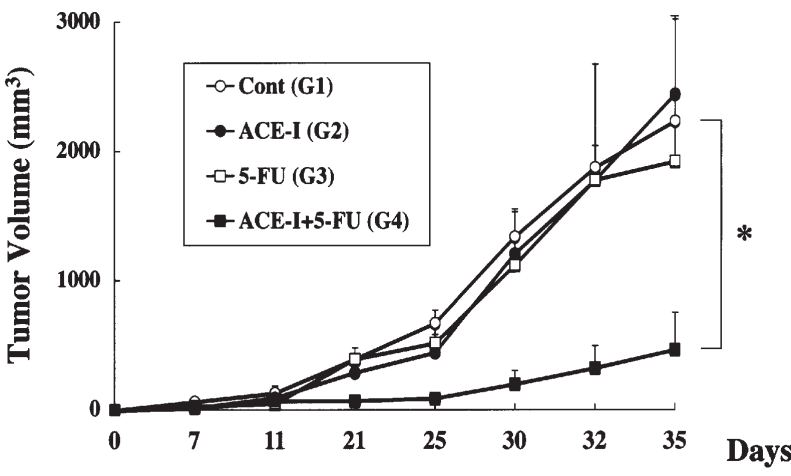

Figure 1. Effects of 5-FU and ACE-I on HCC growth. Group 1 (G1) is the untreated control group $(\bullet)$. The animals in $\mathrm{G} 2(\bullet)$ and G3 ( $\square$ ) received ACE-I and 5 -FU at doses of $0.5 \mathrm{mg} / \mathrm{kg} /$ day and $5 \mathrm{mg} / \mathrm{kg} /$ day by daily gavage, respectively. The group that received combination treatment of ACE-I and 5-FU was designed as G4 (ם). Neither single-agent treatment of ACE-I (G2) nor 5-FU (G3) at low doses showed marked inhibitory effects on the HCC growth as compared to the control group (G1). However, the mice treated with low doses of 5-FU and ACE-I in combination (G4) showed a significant decrease in the tumor volume. The tumor volume was determined by calipers at the indicated time points. Each point represents the mean \pm SD $(n=10)$. * Statistically significant differences between the indicated experimental groups $(\mathrm{p}<0.01)$.

end labeling [terminal deoxynucleotidyl transferase-mediated dUTP nick labeling (TUNEL)] using paraffin-embedded sections. The TUNEL-positive cells were counted using a light microscope. In each tumor, the positive cells in 30 highpower fields at a magnification of $\mathrm{x} 400$ were examined as described previously. For detection of the preneoplastic lesions, anti-GST-P (glutathione S-transferase placental form) antibody (Medical Biological Laboratories Co., Nagoya, Japan) was used as described previously. (29) These immunopositive cells and GST-P-positive preneoplastic lesions were evaluated with Adobe Photoshop and NIH image software as described elsewhere (30).

In vitro and angiogenesis assay. The in vitro angiogenesis was assessed as formation of capillary-like structures of HUVEC co-cultured with human diploid fibroblasts as described previously (31). The experimental procedure followed the instructions provided with the angiogenesis kit (Kurabo, Tokyo). Briefly, the cells were treated with 5-FU and/or ACE-I (perindoprilat) at $1 \times 10^{-7} \mathrm{M}$ on day 1 , and the medium was replaced on days 4,7 , and 9 . On day 11 , the cells were fixed and HUVEC were stained using an anti-human CD31 antibody (Kurabo) according to the protocol provided with the kit. Computer-assisted quantitation of tubule formation was performed in the same way as for the in vivo assay.

Statistical analysis. To assess the statistical significance of the inter-group differences in the quantitative data, Bonferroni's multiple comparison test was used after one-way analysis of variance (ANOVA). This was followed by Barlett's test to determine the homology of variance.

\section{Results}

Effects of 5-FU and ACE-I on HCC growth. We first examined the effects of 5-FU and ACE-I on HCC growth. As shown in
Table I. Effect of 5-FU and/or ACE-I on CD31 mRNA and apoptosis positive cells in the tumors.

\begin{tabular}{lcccc}
\hline & Control & ACE-I & 5-FU & ACE-I+5-FU \\
\hline CD31 mRNA & $0.87^{\mathrm{a}}$ & 0.67 & 0.58 & 0.18 \\
$\begin{array}{l}\text { Apoptotic } \\
\text { index }\end{array}$ & $7.00 \pm 3.891^{\mathrm{b}}$ & $8.83 \pm 6.63$ & $8.50 \pm 3.71$ & $14.23 \pm 5.10^{\mathrm{c}}$ \\
\hline
\end{tabular}

${ }^{\mathrm{a}}$ Gene expression presented after normalization with the glyceraldehyde-3phosphate dehydrogenase internal control. b Data represent means \pm SD. ${ }^{\mathrm{c}}$ Statistically significant differences from control group $(\mathrm{p}<0.01)$.

Fig. 1, single treatment with either ACE-I (G2) or 5-FU (G3) at low doses did not show any marked inhibitory effects on HCC growth as compared to the control group (G1). However, the mice treated with the combination of 5-FU and ACE-I (G4) showed a significant decrease in the tumor volume $(\mathrm{p}<0.01)$. Both compounds at the current doses did not affect the healthy state parameters; e.g. the body weight during the experiment. High dose of 5-FU (50 mg/kg/day) inhibited the HCC growth similar to that of G4, but the mice in this group showed a marked body weight loss as compared with the mice of the other groups, probably because of the cytotoxicity of high dose of 5-FU (data not shown).

Neovascularization and apoptosis in the tumor. We next examined the effects of 5-FU and ACE-I on neovascularization in the tumor to elucidate whether or not the inhibitory effects of these agents are associated with alteration of angiogenesis. We performed a preliminary immunohistochemical analysis of the CD31 on sections from all experimental groups, and found that the combination treatment with 5-FU and ACE-I significantly suppressed the CD31-positive vessels. However, it was hard to accurately evaluate the CD31-positive cells because of difficulties in identifying the little slit vessels in the combination treatment group (data not shown). Hence, we performed a semi-quantitative RT-PCR analysis of the CD31 gene expression to evaluate neovascularization. As shown in Table I, the expression of CD31 in the combination treatment group (G4) was markedly suppressed as compared to the control group $(\mathrm{G} 1)$, whereas neither single treatment with low dose of ACE-I or 5-FU exerted such an inhibitory effect (G2 and G3, respectively). We also examined the effect of 5-FU and ACE-I on apoptosis in the tumor. There were more TUNEL-positive cells in the tumor in the combination treatment of 5-FU and ACE-I than in the groups of 5-FU- or ACE-I-treated animals $(\mathrm{p}<0.01)$. The incidence of apoptosis in the tumor almost corresponded to the effect of tumor development inhibition (Table I).

Expression of VEGF in the tumor. To elucidate whether the combination treatment of 5-FU and ACE-I suppressed the VEGF expression, we measured the VEGF expression in the tumor. Similar to the results of the tumor growth, single treatment with either ACE-I (G2) or 5-FU (G3) at low doses did not show any marked inhibitory effects on the VEGF expression as compared with the control group (G1). The 


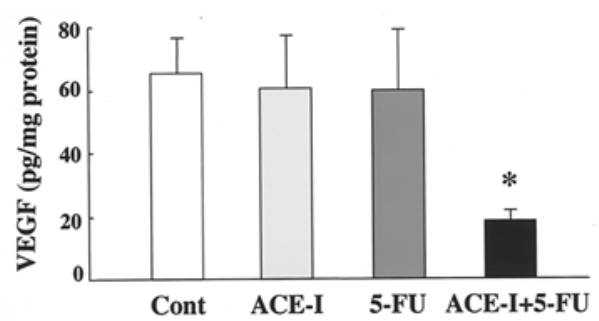

Figure 2. Effects of ACE-I and 5-FU on the VEGF expression in the tumor. The combination treatment of ACE-I and 5-FU exerted a more marked inhibition of the VEGF in the tumor than either single agent treatment $(\mathrm{p}<0.01)$. Each point represents the mean $\pm \mathrm{SD}(\mathrm{n}=5)$. * Statistically significant differences as compared with the control group $(\mathrm{p}<0.01)$. Cont: untreated control group (G1). ACE-I, 5-FU: ACE-I (G2) and 5-FU-treated (G3) group, respectively. ACE-I+5-FU: ACE-I and 5-FU combination-treated group (G4).

expression of VEGF in the combination treatment group (G4) was markedly suppressed as compared to the control group (G1) (Fig. 2). We also examined other angiogenic factors such as bFGF and IL-8, and found that there were no significant differences among these factors following treatment with 5-FU and ACE-I (data not shown).

Effects of 5-FU and ACE-I on the in vitro angiogenesis. We also investigated the in vitro EC tubule formation in the presence or absence of 5-FU and/or ACE-I. We found that neither 5-FU nor ACE-I alone at low dose inhibited the EC tubule formation, whereas the combination treatment of 5-FU and ACE-I significantly suppressed the EC tubule formation (Fig. 3A). Our semi-quantitative analysis showed that the total length of tubules formed in the combination treatment of 5-FU- and ACE-I-treated cultures was significantly less than in the untreated control culture $(\mathrm{p}<0.01)$ (Fig. 3B).
Table II. Details of experimental groups administered DEN with ACE-I and/or 5-FU.

\begin{tabular}{lccc}
\hline & $\begin{array}{c}\text { Final body } \\
\text { weight }(\mathrm{g})\end{array}$ & $\begin{array}{c}\text { Relative liver weight } \\
(\mathrm{g} / 100 \text { g body weight })\end{array}$ & $\begin{array}{c}\text { ALT } \\
(\mathrm{U} / \mathrm{l})\end{array}$ \\
\hline DEN & $245 \pm 17^{\mathrm{a}}$ & $2.41 \pm 0.42$ & $224.4 \pm 37.6$ \\
+ ACE-I & $252 \pm 20$ & $2.46 \pm 0.33$ & $234.3 \pm 41.3$ \\
+5 -FU & $257 \pm 19$ & $2.54 \pm 0.29$ & $240.8 \pm 36.5$ \\
+ ACE-I+5-FU & $254 \pm 22$ & $2.44 \pm 0.28$ & $235.7 \pm 40.3$ \\
\hline
\end{tabular}

a Data represent means $\pm \mathrm{SD}$.

Effects of 5-FU and ACE-I against hepatocarcinogenesis. In the hepatocarcinogenesis experiment, all rats survived throughout the experiment. Neither the body weight nor the liver weights changed in any of the experimental groups. Low doses of 5FU and ACE-I did not cause any alteration in the serum ALT levels (Table II). Neither ascites nor organ abnormalities were observed at the end of the experiment in any of the groups (data not shown). No histological changes indicating liver injury or liver fibrosis development were observed, except for the development of GST-P-positive preneoplastic lesions. As shown in Fig. 4, different from the HCC growth, single treatment with ACE-I suppressed the development of preneoplastic lesions as compared with the control group $(\mathrm{p}<0.01)$, whereas 5-FU did not. The combination treatment of both agents exerted a more potent inhibitory effect as compared with the ACE-I-treated group $(\mathrm{p}<0.01)$. No positive preneoplastic lesions were found in the PBS-treated group (G5).

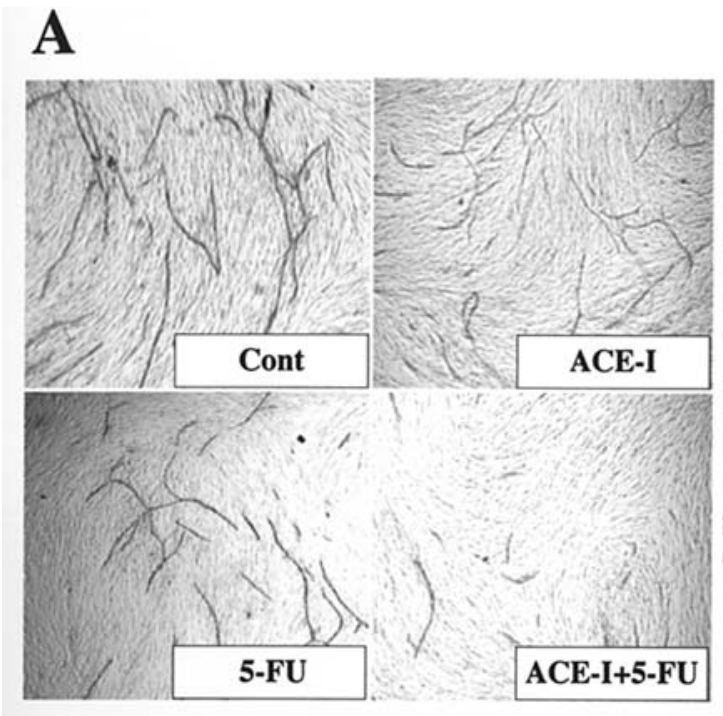

B

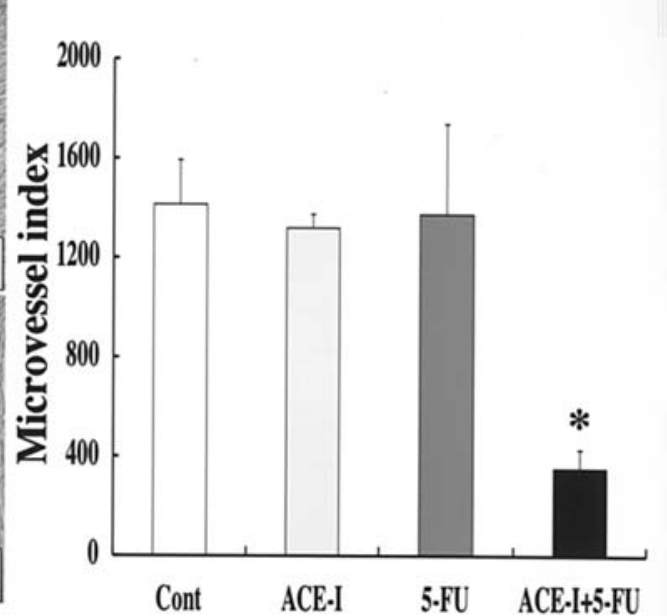

Figure 3. Effects of ACE-I and 5-FU on the in vitro EC tubular formation. (A) ACE-I alone and 5-FU alone did not inhibit the EC tubule formation. However, the combination treatment of ACE-I and 5-FU markedly attenuated the EC tubule formation. (B) Computer-assisted quantitative analysis showed that the total length of tubules formed in the ACE-I- or 5-FU-treated cultures was significantly less than in the untreated control culture. The combination treatment of ACE-I and 5-FU resulted in a marked inhibition of the EC tubular formation than either single-agent treatment. The total tubule length was measured by an image analysis system as described in the Materials and methods. The data represent the mean \pm SD $(n=6)$. "Statistically significant differences between the indicated experimental groups $(\mathrm{p}<0.01)$. Cont: untreated control group. ACE-I, 5-FU: ACE-I and 5-FU-treated group, respectively. ACE-I+5-FU: ACE-I and 5 -FU combination-treated group. 
A
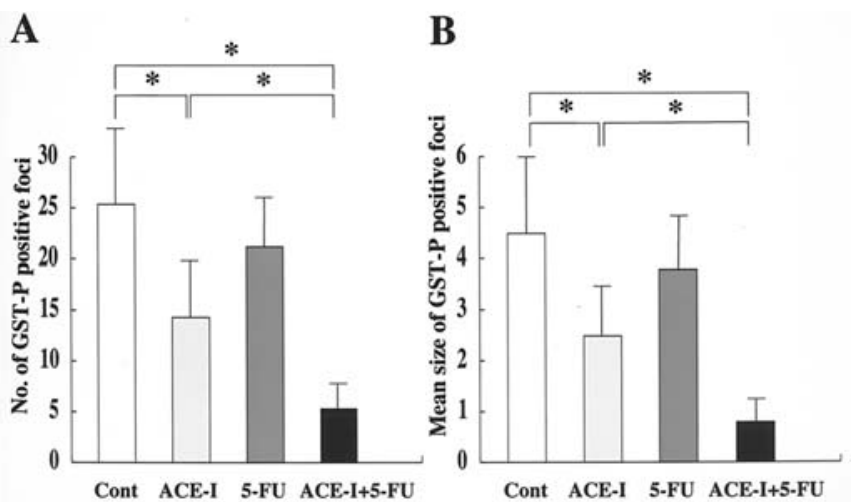

Figure 4. Effects of ACE-I and 5-FU on the development of DEN-induced GST-P-positive preneoplastic lesions. The number (A) and size (B) of the GST-P-positive lesions were suppressed by the treatment of ACE-I but not 5-FU. The combination treatment of ACE-I and 5-FU exerted a significant inhibitory effect as compared with that of ACE-I. No positive preneoplastic lesions were found in the PBS-treated group. Each point represents the mean \pm SD $(n=10) .{ }^{*}$ Statistically significant differences between the indicated experimental groups $(\mathrm{p}<0.01)$. Cont: untreated control group $(\mathrm{G} 1)$. ACE-I, 5-FU: ACE-I (G2) and 5-FU-treated (G3) group, respectively. ACE-I+5-FU: ACE-I and 5-FU combination-treated group (G4). PBS: PBS-treated negative control group (G5).

\section{Discussion}

It is now widely recognized that the growth of any solid tumor depends on angiogenesis. Without angiogenesis, the tumor ceases to grow even beyond a few millimeters in size; i.e. 'tumor dormancy' $(2,32)$. Since inhibition of angiogenesis is now considered to be a promising approach in cancer therapy, efforts are currently directed worldwide at overcoming tumor angiogenesis. In animal models, anti-angiogenic compounds have proven very successful in inhibiting tumor development. These agents have exhibited a reduced toxicity and a decreased likelihood of causing development of drug resistance as compared to the conventional chemotherapeutic agents at the maximum tolerable high doses (2,32). Since emerging evidence has demonstrated that angiogenesis plays a pivotal role in $\mathrm{HCC}$, an anti-angiogenic therapy would be a fruitful approach against $\mathrm{HCC}$.

In the current study, we employed an ACE-I as an antiangiogenic agent. A retrospective cohort study on 5207 patients receiving ACE-I or other anti-hypertensive drugs with a 10year follow-up demonstrated that ACE-I treatment may decrease the incidence of adult cancer and fetal cancer (33). The other anti-hypertensive drugs, e.g. calcium channels blockers, diuretics, and B-blockers, have no apparent effects on the risk of cancer development. AT-II has been shown to induce neovascularization in experimental models both in vitro and in vivo, and it also increased the VEGF expression. (34) These pro-angiogenic effects were attenuated by treatment with ACE-I. (35) We previously reported that ACE-I could suppress the HCC growth along with inhibition of neovascularization and VEGF expression $(22,36)$. In the current study, however, we did not observe any anti-tumoral effect with the single treatment of ACE-I, which seemed to be contradictory to our previous reports $(22,37,38)$. The dose $(0.5 \mathrm{mg} / \mathrm{kg})$ in the current study was much lower than in the previous reports $(2-8 \mathrm{mg} / \mathrm{kg})$. It has been reported that the inhibitory effect of ACE-I on the AT-II-induced angiogenesis is dose-dependent (22). Moreover, ACE-I at a very low dose $\left(1 \times 10^{-7} \mathrm{M}\right)$ in the current study did not inhibit the EC tubular formation, whereas ACE-I at $1 \times 10^{-6} \mathrm{M}$ exerted significant suppression $(39,40)$. It could be possible that the dose in this study did not reach the threshold level to exert an antitumoral effect via anti-angiogenesis. In addition to HCC growth inhibition, we observed that the combination treatment of ACE-I and 5-FU significantly suppressed hepatocarcinogenesis. Contrary to the HCC growth experiment, single treatment with low-dose ACE-I inhibited the development of GST-P-positive lesions although the magnitude was not potent as compared with the combination treatment of 5-FU and ACE-I. It has been reported that the anti-angiogenic strategy would be more effective in the early stage rather than in the late stage of tumor development (41). It could be possible that the anti-angiogenic activity of a low dose of ACE-I could affect the early carcinogenesis process but not the already transformed tumor growth. Further studies are required to elucidate the mechanism in the future.

5 -FU is an important chemotherapeutic drug with an established role in cancer treatment, especially for hepatogastrointestinal tumors (42). As well as ACE-I, a low dose of 5-FU did not show any significant inhibitory effect on the HCC growth. We observed that the low dose of 5-FU did not show any inhibitory effect on proliferation of the HCC cells in vitro, suggesting that the direct cytotoxic effect on the tumor cells was not the main mechanism of the anti-tumoral effect of 5-FU in the current study (data not shown). Recent studies have revealed that the conventional cytotoxic chemotherapeutic drugs also have anti-angiogenic effects at small doses, which could contribute to their anti-tumoral efficacy (5). The in vitro and in vivo assays demonstrated that 5-FU exerted anti-angiogenic activity in a dose-dependent manner (43). It has been reported that combination treatments of antiangiogenic agent and conventional chemotherapeutic drugs at low doses exert a synergistic potent inhibitory effect on the tumor development via anti-angiogenic activity (4). We also observed that the combination treatment of ACE-I and 5-FU exerted a significant inhibitory effect on EC tubular formation, indicating that the anti-tumoral effect of this combination regimen may be, at least partly, mediated by synergistic antiangiogenic activities.

It has been shown that treatment with anti-angiogenic agents induced a marked increase of apoptosis in the tumor, whereas it did not alter the tumor cell proliferation itself (44). In this study, immunohistochemical analysis by TUNEL assay revealed that the combination treatment with ACE-I and 5-FU significantly increased apoptosis in the tumor, whereas our in vitro study showed that the tumor cell proliferation was not altered by either single or combination treatment with ACE-I and 5-FU (data not shown). On the contrary, with both agents, the EC tubular formation was significantly suppressed. These findings, taken together, suggest that ACE-I and 5-FU reduced the tumor growth via inhibition of EC tubular formation, which in turn may induce apoptosis of the tumor cells, rather than via direct inhibition of the tumor cell proliferation.

In summary, we have shown here that the combination treatment of ACE-I and 5-FU significantly inhibited HCC 
growth along with suppression of angiogenesis and VEGF expression. This combination also inhibited early hepatocarcinogenesis. Since both agents are currently widely used in the clinical practice, this combination regimen may represent a potential new strategy against HCC in the future.

\section{References}

1. Carmeliet P: Angiogenesis in health and disease. Nat Med 9: 653-660, 2003.

2. Carmeliet P and Jain RK: Angiogenesis in cancer and other diseases. Nature 407: 249-257, 2000.

3. Saaristo A, Karpanen T and Alitalo K: Mechanisms of angiogenesis and their use in the inhibition of tumor growth and metastasis. Oncogene 19: 6122-6129, 2000.

4. Kerbel RS: Clinical trials of antiangiogenic drugs: opportunities, problems, and assessment of initial results. J Clin Oncol 19: 45S-51S, 2001.

5. Kerbel RS and Kamen BA: The anti-angiogenic basis of metronomic chemotherapy. Nat Rev Cancer 4: 423-436, 2004.

6. Davis DW, Inoue K, Dinney CP, Hicklin DJ, Abbruzzese JL and McConkey DJ: Regional effects of an antivascular endothelial growth factor receptor monoclonal antibody on receptor phosphorylation and apoptosis in human $253 \mathrm{~J} \mathrm{B-V}$ bladder cancer xenografts. Cancer Res 64: 4601-4610, 2004.

7. Scappaticci FA, Smith R, Pathak A, et al: Combination angiostatin and endostatin gene transfer induces synergistic antiangiogenic activity in vitro and antitumor efficacy in leukemia and solid tumors in mice. Mol Ther 3: 186-196, 2001.

8. Ferrara N and Kerbel RS: Angiogenesis as a therapeutic target. Nature 438: 967-974, 2005.

9. Gasparini G, Longo R, Fanelli M and Teicher BA: Combination of antiangiogenic therapy with other anticancer therapies: results, challenges, and open questions. J Clin Oncol 23: 1295-1311, 2005.

10. Befeler AS and Di Bisceglie AM: Hepatocellular carcinoma: diagnosis and treatment. Gastroenterology 122: 1609-1619, 2002.

11. Mise M, Arii S, Higashituji H, et al: Clinical significance of vascular endothelial growth factor and basic fibroblast growth factor gene expression in liver tumor. Hepatology 23: 455-464, 1996.

12. Yamaguchi R, Yano H, Iemura A, Ogasawara S, Haramaki M and Kojiro M: Expression of vascular endothelial growth factor in human hepatocellular carcinoma. Hepatology 28: 68-77, 1998.

13. Yoshiji H, Kuriyama S, Hicklin DJ, et al: KDR/Flk-1 is a major regulator of vascular endothelial growth factor-induced tumor development and angiogenesis in murine hepatocellular carcinoma cells. Hepatology 30: 1179-1186, 1999.

14. Yoshiji H, Kuriyama S, Yoshii J, et al: Involvement of the vascular endothelial growth factor receptor-1 in murine hepatocellular carcinoma development. J Hepatol 41: 97-103, 2004.

15. Yoshiji H, Kuriyama S, Yoshii J, et al: Vascular endothelial growth factor tightly regulates in vivo development of murine hepatocellular carcinoma cells. Hepatology 28: 1489-1496, 1998.

16. Li CY, Shan S, Huang Q, et al: Initial stages of tumor cellinduced angiogenesis: evaluation via skin window chambers in rodent models. J Natl Cancer Inst 92: 143-147, 2000.

17. Bergers G, Javaherian K, Lo KM, Folkman J and Hanahan D: Effects of angiogenesis inhibitors on multistage carcinogenesis in mice. Science 284: 808-812, 1999.

18. Brandvold KA, Neiman P and Ruddell A: Angiogenesis is an early event in the generation of myc-induced lymphomas. Oncogene 19: 2780-2785, 2000.

19. Bolontrade MF, Stern MC, Binder RL, Zenklusen JC, GimenezConti IB and Conti CJ: Angiogenesis is an early event in the development of chemically induced skin tumors. Carcinogenesis 19: 2107-2113, 1998.

20. Frachon S, Gouysse G, Dumorti J, et al: Endothelial cell marker expression in dysplastic lesions of the liver: an immunohistochemical study. J Hepatol 34: 850-857, 2001.

21. Yoshiji H, Kuriyama S, Yoshii J, et al: Halting the interaction between vascular endothelial growth factor and its receptors attenuates liver carcinogenesis in mice. Hepatology 39: 1517-1524, 2004.

22. Yoshiji H, Kuriyama S, Kawata M, et al: The angiotensin-iconverting enzyme inhibitor perindopril suppresses tumor growth and angiogenesis: possible role of the vascular endothelial growth factor. Clin Cancer Res 7: 1073-1078, 2001.
23. Yoshiji H, Kuriyama S, Noguchi R, et al: Combination of vitamin $K(2)$ and the angiotensin-converting enzyme inhibitor, perindopril, attenuates the liver enzyme-altered preneoplastic lesions in rats via angiogenesis suppression. J Hepatol 42: 687-693, 2005.

24. Yoshiji H, Kuriyama S, Ways DK, et al: Protein kinase C lies on the signaling pathway for vascular endothelial growth factormediated tumor development and angiogenesis. Cancer Res 59: 4413-4418, 1999.

25. Yoshiji H, Nakae D, Kinugasa T, et al: Inhibitory effect of dietary iron deficiency on the induction of putative preneoplastic foci in rat liver initiated with diethylnitrosamine and promoted by phenobarbital. Br J Cancer 64: 839-842, 1991.

26. Shiota G, Harada K, Ishida M, et al: Inhibition of hepatocellular carcinoma by glycyrrhizin in diethylnitrosamine-treated mice. Carcinogenesis 20: 59-63, 1999.

27. Shibuya M: Structure and function of VEGF/VEGF-receptor system involved in angiogenesis. Cell Struct Funct 26: 25-35, 2001.

28. Yoshiji H, Kuriyama S, Yoshii J, et al: Synergistic effect of basic fibroblast growth factor and vascular endothelial growth factor in murine hepatocellular carcinoma. Hepatology 35 : 834-842, 2002.

29. Yoshiji H, Kuriyama S, Yoshii J, et al: The copper-chelating agent, trientine, attenuates liver enzyme-altered preneoplastic lesions in rats by angiogenesis suppression. Oncol Rep 10: 1369-1373, 2003.

30. Yoshiji H, Yoshii J, Ikenaka Y, et al: Inhibition of reninangiotensin system attenuates liver enzyme-altered preneoplastic lesions and fibrosis development in rats. J Hepatol 37: 22-30, 2002.

31. Saito M, Hamasaki $\mathbf{M}$ and Shibuya $\mathbf{M}$ : Induction of tube formation by angiopoietin-1 in endothelial cell/fibroblast coculture is dependent on endogenous VEGF. Cancer Sci 94: 782-790, 2003.

32. Kerbel RS: Tumor angiogenesis: past, present and the near future. Carcinogenesis 21: 505-515, 2000.

33. Lever AF, Hole DJ, Gillis CR, et al: Do inhibitors of angiotensin-I-converting enzyme protect against risk of cancer? Lancet 352: 179-184, 1998 .

34. Yoshiji H, Kuriyama S and Fukui H: Angiotensin-I-converting enzyme inhibitors may be an alternative anti-angiogenic strategy in the treatment of liver fibrosis and hepatocellular carcinoma. Possible role of vascular endothelial growth factor. Tumour Biol 23: 348-356, 2002.

35. Yoshiji H, Kuriyama S and Fukui H: Perindopril: possible use in cancer therapy. Anticancer Drugs 13: 221-228, 2002.

36. Noguchi R, Yoshiji H, Kuriyama S, et al: Combination of interferon-beta and the angiotensin-converting enzyme inhibitor, perindopril, attenuates murine hepatocellular carcinoma development and angiogenesis. Clin Cancer Res 9: 6038-6045, 2003.

37. Yoshiji H, Kuriyama S, Noguchi R and Fukui H: Angiotensin-I converting enzyme inhibitors as potential anti-angiogenic agents for cancer therapy. Curr Cancer Drug Targets 4: 555-567, 2004.

38. Yoshiji H, Kuriyama S, Yoshii J, et al: Angiotensin-II type 1 receptor interaction is a major regulator for liver fibrosis development in rats. Hepatology 34: 745-750, 2001.

39. Yoshiji H, Noguchi R, Kuriyama S, Yoshii J and Ikenaka Y: Combination of interferon and angiotensin-converting enzyme inhibitor, perindopril, suppresses liver carcinogenesis and angiogenesis in mice. Oncol Rep 13: 491-495, 2005.

40. Yoshiji H, Noguchi R, Kuriyama S, et al: Suppression of renin-angiotensin system attenuates hepatocarcinogenesis via angiogenesis inhibition in rats. Anticancer Res 25: 3335-3340, 2005.

41. Carmeliet P: Angiogenesis in life, disease and medicine. Nature 438: 932-936, 2005.

42. Soong R and Diasio RB: Advances and challenges in fluoropyrimidine pharmacogenomics and pharmacogenetics. Pharmacogenomics 6: 835-847, 2005.

43. Basaki Y, Chikahisa L, Aoyagi K, et al: gamma-Hydroxybutyric acid and 5-fluorouracil, metabolites of UFT, inhibit the angiogenesis induced by vascular endothelial growth factor. Angiogenesis 4: 163-173, 2001.

44. Yoshii J, Yoshiji H, Kuriyama S, et al: The copper-chelating agent, trientine, suppresses tumor development and angiogenesis in the murine hepatocellular carcinoma cells. Int J Cancer 94: 768-773, 2001. 